\title{
Fatores de risco para mortalidade infantil pós-neonatal
}

\author{
Risk factors for post-neonatal infant mortality
}

Paulo Eduardo A. Baldin', Paulo Cesar K. Nogueira²

\section{RESUMO}

Objetivo: Verificar, dentre os critérios do Programa do Recém-Nascido de Risco da Secretaria de Saúde de Santos, São Paulo, os fatores de risco para a mortalidade pós-neonatal.

Métodos: Estudo retrospectivo de dados secundários de 22.452 fichas coletadas ao nascimento, de janeiro de 1998 a dezembro de 2001. A variável dependente foi o óbito pósneonatal. As variáveis independentes foram: peso ao nascer $<2.500 \mathrm{~g}$, malformação ao nascer, necessidade de internação do recém-nascido após a alta materna, gravidez indesejada, chefe de família desempregado, irmão <2 anos e mãe sem companheiro. A análise estatística foi feita por regressão logística.

Resultados: Durante o período do estudo, dentre as 22.452 crianças incluídas no estudo, 97 faleceram entre 29 dias e um ano de idade. $\mathrm{Na}$ análise bivariada, foram indicadores de óbito: "peso <2.500g", "malformação ao nascer", "internação ao nascer", "chefe familiar desempregado", “irmão <2 anos" e "mãe sem companheiro". Na análise multivariada por regressão logística, as variáveis "peso ao nascer < 2.500g", "malformação ao nascer", "necessidade de internação do recém-nascido após a alta materna” e "irmão menor de dois anos no domicílio" se associaram significantemente ao óbito infantil pós-neonatal.

Conclusões: Os critérios utilizados pelo Programa do Recém-Nascido de Risco foram capazes de identificar crianças de risco para óbito pós-neonatal.

Palavras-chave: fatores de risco; mortalidade pósneonatal; criança.

\section{ABSTRACT}

Objective: To assess, among the criteria adopted by the Program of Neonates at Risk of the Health Department of the Municipality of Santos, São Paulo, Brazil, the risk factors for post-neonatal infant mortality.

Methods: In a retrospective study, data from 22,452 newborn charts, from 1998 to 2001, were retrieved. Logistic regression was applied to detect risk factors for the dependent variable: death between 29 days and one year old. The independent variables were: birth weight $<2,500 \mathrm{~g}$, congenital malformation, unwanted pregnancy, need of hospitalization after mother's discharge, unemployed house chief, siblings $<2$ years old and single mother.

Results: During the studied period, among the 22,452 children, there were 97 deaths of children between 29 days and one year old. In the bivariate analysis, the variables "birth weight <2,500g", "congenital malformations", "need of hospitalization after mother's discharge", "unemployed house chief", "siblings < 2 years" and "single mother" were significantly associated to post-neonatal death. By logistic regression analysis, "birth weight $<2.500 \mathrm{~g}$ ", "congenital malformations", "need of hospitalization after mother's discharge" and "siblings <2 years old" were significantly associated to post-neonatal mortality.

Conclusions: The criteria adopted by the Program of Neonates at Risk were useful to identify infants at risk for death from 29 days to one year of life.

Key-words: risk factors; postneonatal mortality; child.
'Mestre em Ciências da Saúde pelo Centro Universitário Lusíada (Unilus), Santos, SP, Brasil

2Professor de Pediatria do curso de Ciências Médicas do Unilus e doutor em Pediatria pela Universidade Federal de São Paulo, São Paulo, SP, Brasil

\author{
Endereço para correspondência: \\ Paulo Eduardo A. Baldin \\ Rua Nabuco de Araújo, 139, apto. 24 \\ CEP 11025-010 - Santos/SP \\ E-mail pbaldin@uol.com.br \\ Recebido em: 27/10/2007 \\ Aprovado em: 10/4/2008
}




\section{Introdução}

Em 1990, o coeficiente de mortalidade infantil no município de Santos, São Paulo, era de 36,1 por mil nascidos vivos. Neste mesmo ano, foi realizada uma busca ativa de todos os atestados de óbitos de crianças menores de um ano de idade residentes na cidade, como ponto de partida para uma revisão criteriosa dos prontuários de internação e dos prontuários das Unidades Básicas de Saúde (UBS), sendo complementada por entrevista junto aos pais das crianças falecidas. Tal esforço culminou com a criação do Programa de Vigilância do Recém-Nascido de Risco, cujo objetivo foi reduzir a mortalidade infantil por meio da atenção diferenciada às crianças com maior risco de morte. A atividade do programa era a avaliação do risco da criança ao nascimento, na maternidade, por meio de uma ficha com critérios específicos. O programa previa atenção especial para os recém-nascidos considerados de risco, com visita domiciliar de rotina e busca ativa, no caso de falta, além de retornos mais freqüentes à UBS onde a criança era acompanhada ${ }^{(1)}$.

Os critérios de identificação do risco ao nascer adotados pelo programa foram baseados naqueles consagrados pela literatura ${ }^{(2-4)}$ e que se mostraram prevalentes, com significância estatística, nos casos de óbito revisados no ano de $1990^{(5-7)}$. Dessa forma, foram considerados os seguintes critérios de risco: peso ao nascer igual ou inferior a $2.500 \mathrm{~g}$, malformações congênitas detectadas ao nascimento, necessidade de internação do recém-nascido após alta materna, gravidez indesejada, chefe de família desempregado no momento do nascimento da criança, irmão menor de dois anos e mãe sem companheiro à época do parto ${ }^{(8-10)}$.

No período de 1998 a 2001, a aplicação do programa teve cobertura elevada e a maioria das fichas de critérios encontravase digitada na base de dados da Secretaria de Saúde de Santos, além de terem sido realizadas revisões de todos os óbitos pósneonatais. Dessa forma, o objetivo dessa pesquisa foi verificar, dentre os critérios adotados pelo Programa de Vigilância do Recém-Nascido de Risco da Secretaria de Saúde de Santos, quais variáveis se associaram à mortalidade infantil pós-neonatal.

\section{Métodos}

Estudo retrospectivo, a partir de dados secundários coletados nas fichas de nascimento do Programa de Vigilância do Recém-Nascido de Risco da Secretaria de Saúde de Santos, no período de janeiro de 1998 a dezembro de 2001.

No período do estudo, foram registrados 25.125 nascidos vivos no município, dos quais 23.629 haviam sido avaliados ao nascer pelo Programa (fichas digitadas). Destas, 288 fichas foram excluídas por tratarem de óbitos neonatais e 889 fichas foram excluídas por mau preenchimento, obtendo-se um total de 22.452 casos válidos (Figura 1).

Durante todo o período de atividade do programa, foram mantidas regularmente as coletas de atestados de óbito junto aos cartórios, além da revisão de prontuários e entrevistas domiciliares com os pais - atividades previstas e realizadas como parte do próprio Programa, o que gerou um banco consistente de informações sobre os óbitos de crianças no período. Tal banco, aliado aos constantes cruzamentos com o total de óbitos registrados na Fundação Sistema Estadual de Análise de Dados (Seade) como parte da atividade de rotina do Programa, tornou remota a possibilidade de perdas. Por meio do banco de dados de fichas dos casos válidos $(\mathrm{n}=22.452)$, foram listados os óbitos pós-neonatais do período sendo, então, criados dois grupos: 97 crianças que faleceram entre 29 dias e um ano de idade e 22.355 sobreviventes.

Para os dois grupos, foram calculadas e analisadas as diferenças de freqüências das seguintes variáveis: peso ao nascer $<2.500 \mathrm{~g}$, malformação ao nascer, necessidade de internação do recém-nascido após a alta materna, gravidez indesejada, chefe de família desempregado, irmão $<2$ anos e mãe sem companheiro.

A análise dos bancos de dados foi realizada por decisão da equipe de Vigilância Epidemiológica, da qual faz parte um dos autores da pesquisa, e a publicação dos resultados foi autorizada e estimulada pela Secretaria da Saúde de Santos.

Para análise estatística, determinou-se a razão de chances (OR) e o intervalo de confiança (IC95\%) de cada variável independente estudada em relação à variável dependente. A seguir, foi realizada a análise multivariada por meio da regressão logística, estudando-se as variáveis independentes associadas

Total de nascimentos no período $=25.125$

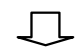

Total de fichas digitadas $=23.629$

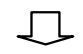

Óbitos de 0 a 28 dias excluídos $=288$

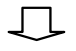

Fichas excluídas por mau preenchimento $=889$

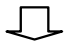

Total de casos válidos $=22.452$

Figura 1 - Fluxograma para inclusão de crianças no estudo. 
Tabela 1 - Análise bivariada das variáveis independentes versus a variável desfecho óbito pós-neonatal

\begin{tabular}{lccrrc}
\hline Variável & $\begin{array}{c}\text { Óbitos } \\
(\mathbf{n = 9 7 )}\end{array}$ & $\begin{array}{c}\text { Sobreviventes } \\
(\mathbf{n = 2 . 3 5 5 )}\end{array}$ & OR & IC95\% & $\boldsymbol{p}$ \\
\hline Malformação ao nascer & $16(16 \%)$ & $191(1 \%)$ & 22,9 & $13,1-39,9$ & $<0,05$ \\
Necessidade de internação ao nascer & $45(46 \%)$ & $1.375(6 \%)$ & 13,2 & $8,8-19,7$ & $<0,05$ \\
Peso ao nascer menor que 2.500g & $38(40 \%)$ & $175(8 \%)$ & 7,5 & $5,0-11,4$ & $<0,05$ \\
Irmão menor de 2 anos & $17(17 \%)$ & $1.544(7 \%)$ & 2,8 & $1,6-4,8$ & $<0,05$ \\
Mãe sem companheiro & $20(20 \%)$ & $2.944(13 \%)$ & 1,6 & $1,0-2,7$ & $<0,05$ \\
Chefe de família desempregado & $28(29 \%)$ & $4.390(19 \%)$ & 1,6 & $1,0-2,5$ & $<0,05$ \\
Gravidez indesejada & $0(0 \%)$ & $5(1 \%)$ & 1,0 & $1,2-1,3$ & NS \\
\hline
\end{tabular}

Tabela 2 - Regressão logística com todas as variáveis significantes após a análise bivariada

\begin{tabular}{lrr}
\hline Variável & OR & IC95\% \\
\hline Malformação ao nascer & 13,4 & $7,3-24,7$ \\
Necessidade de internação & 6,5 & $4,0-10,6$ \\
ao nascer & & \\
Peso ao nascer menor & 2,9 & $1,7-4,8$ \\
que 2.500g & 2,7 & $1,5-4,7$ \\
Irmão menor de 2 anos & 1,1 & $0,6-1,9$ \\
Mãe sem companheiro & 1,1 & $0,7-1,8$ \\
\hline
\end{tabular}

significantemente ao evento óbito pós-neonatal, com a entrada manual de variável a variável no modelo. Para todos os cálculos estatísticos, utilizou-se o nível de $5 \%$ para rejeição da hipótese de nulidade.

\section{Resultados}

Foram analisadas 22.452 fichas de crianças que obedeceram aos critérios de inclusão no estudo. Do total de crianças estudadas, 22.355 sobreviveram e 97 evoluíram para óbito entre 29 dias e um ano de idade.

Entre os nascidos vivos, $11.533(51,4 \%)$ crianças eram do sexo masculino, observando-se proporção semelhante de óbitos pós-neonatais em ambos os sexos (masculino:53/97=54,7\%). O parto cesáreo ocorreu em $11.533(51,4 \%)$ casos estudados.

A partir da análise bivariada, observou-se associação estatística significante entre óbito pós-neonatal e todas as variáveis estudadas, exceto gravidez indesejada (Tabela 1).

Quando se aplicou a regressão logística, as variáveis "peso ao nascer inferior a $2.500 \mathrm{~g}$ ", "malformação ao nascer", "necessidade de internação do recém-nascido após a alta materna" e “irmão <2 anos" se associaram de forma significante à mortalidade pós-neonatal. Já as variáveis "mães sem companheiro" e "chefe de família desempregado" deixaram de ser significantes (Tabela 2).

\section{Discussão}

Segundo a Fundação Seade, o coeficiente de mortalidade infantil para o município de Santos, em 1990, era de 36,1 por mil nascidos vivos, sendo os componentes neonatal e pósneonatal respectivamente 22,6 por mil e 13,4 por mil. Em 2001, o coeficiente de mortalidade infantil diminuiu para 16,9 por mil nascidos vivos, com índices de 10,8 por mil e 6,1 por mil para os componentes neonatal e pós-neonatal, respectivamente ${ }^{(11)}$.

Apesar de, no presente estudo, existirem associações estatísticas entre diversas variáveis independentes e o evento óbito pós-neonatal, é impossível atribuir uma relação de causa e efeito entre a aplicação do programa e a redução da mortalidade ${ }^{(12)}$. No entanto, é possível que o programa de detecção do recém-nascido de risco tenha colaborado para a maior atenção dada a tais crianças e, por conseguinte, tenha contribuído de maneira decisiva para a redução da mortalidade infantil.

Para a variável "malformação ao nascer", foram consideradas quaisquer alterações físicas, inclusive as de menor importância. Esta variável se associou estatisticamente ao óbito pós-neonatal, indicando a necessidade de seguimento de crianças portadoras de malformações ${ }^{(13,14)}$. Chama atenção que, tanto na análise bivariada quanto na multivariada, a malformação foi o fator que, quando presente, indicou maior chance de óbito. No entanto, é possível ter havido subnotificação de malformações simples, que podem ter passado despercebidas no preenchimento da ficha. Nesse caso, o valor de tal achado deve ser atribuído ao impacto de malformações graves na mortalidade pós-neonatal, da mesma forma que se observa na mortalidade neonatal ${ }^{(15)}$.

Outra variável com comportamento semelhante na casuística estudada foi a necessidade de internação do recémnascido após a alta materna. Todas as internações foram consideradas, independentemente do diagnóstico e do tempo de duração das mesmas. Mais uma vez, o significado 
deste achado deve estar relacionado a patologias complexas do período neonatal. De qualquer forma, a informação de que a criança permaneceu internada é fácil de ser obtida e, associada ao fato de ser estatisticamente relacionada ao óbito pós-neonatal, pode representar mais um fator de alerta importante a ser considerado na atenção à criança.

Desde os estudos de Barros $e t$ al $l^{(6)}$, que demonstraram forte relação estatística do baixo peso ao nascer e a mortalidade infantil, inúmeros trabalhos vêm confirmando este achado. A taxa de baixo peso ao nascer de nosso estudo foi de $8 \%$ e está dentro dos limites aceitos pela Organização Mundial da Saúde. Apesar disso, houve associação significante entre o baixo peso ao nascer e o óbito pós-neonatal, nos mesmos níveis dos encontrados na literatura (OR 2,9; IC95\% 1,7 a 4,8$)^{(16-19)}$.

É mais difícil investigar a relação entre as variáveis socioeconômicas, como gravidez indesejada, mãe sem companheiro e chefe de família desempregado, e o óbito pós-neonatal. Entretanto, algumas variáveis são subjetivas e de difícil demonstração (por exemplo, gravidez indesejada). Além disso, não houve nenhum óbito no grupo da gravidez indesejada e a frequiência de tal variável na amostra total foi muito baixa. Já o comportamento dos outros fatores é muito dinâmico, por exemplo, mãe sem companheiro e chefe de família desempregado ${ }^{(20,21)}$. Talvez por isso, em nossos dados, a associação entre óbito pós-neonatal e mãe sem companheiro ou chefe de família desempregado tenha sido significante na análise bivariada e não significante na regressão logística.
Tal fato pode sugerir que o papel dessas variáveis pode ser menor ou de difícil avaliação com o método de pesquisa adotado. No entanto, a existência de irmão menor de dois anos no domicílio mostrou associação significante com o óbito nas duas análises realizadas, sugerindo que esse fator realmente tem importância como discriminador independente de risco para o desfecho estudado.

A natureza retrospectiva e o embasamento em dados secundários são as maiores limitações metodológicas deste estudo, o que acabou por circunscrever nossa análise aos fatores que constam da ficha do programa. Entretanto, o presente estudo não teve o objetivo de buscar novas variáveis, mas de confirmar a aplicabilidade das variáveis adotadas num programa já em atividade, sendo de interesse o exercício de papel crítico do mesmo, inclusive para reprodução do método em outros municípios com altas taxas de mortalidade pós-neonatal. Dessa forma, antes de concluir acerca do risco de forma definitiva, este estudo aponta quais fatores, dentre os atualmente conhecidos, mais influenciaram a mortalidade pós-neonatal.

Pôde-se concluir que o método de determinação de fatores de risco ao nascer é eficaz para prever o óbito pósneonatal e deve ser aplicado como coadjuvante no controle da mortalidade infantil. Além disso, as variáveis "peso ao nascer igual ou inferior a $2.500 \mathrm{~g}$ ", "malformação ao nascer", "necessidade de internação do recém-nascido após a alta materna" e "irmão menor de dois anos no domicílio" se associaram significantemente ao óbito pós-neonatal.

\section{Referências bibliográficas}

1. Prefeitura Municipal de Santos, Secretaria de Higiene e Saúde, Departamento do Centro de Controle de Doenças. Normas do programa de vigilância do recém-nascido de risco. 1991. D.O. 200 n. 21, seção 1:24-25. Santos (Brasil): Prefeitura Municipal de Santos; 1996.

2. Victora CG, Barros FC, Vaughan JP, editores. Epidemiologia da desigualdade: um estudo longitudinal de 6.000 crianças brasileiras. São Paulo: Hucitec; 1988.

3. Shimakura SE, Carvalho MS, Aerts DR, Flores R. Spatial risk distribution: modeling infant mortality in Porto Alegre, Rio Grande do Sul State, Brazil. Cad Saude Publica 2001;17:1251-61.
4. França E, Moreira de Souza J, Crosland Guimarães MD, Goulart EM, Colosimo E, de Figueiredo Antunes CM. Association between socioeconomic factors and infant deaths due to diarrhea, pneumonia, and malnutrition in a metropolitan area of Southeast Brazil: a case-control study. Cad Saude Publica 2001;17:1437-47.

5. Victora CG, Barros, FC, Halpern R, Menezes AM, Horta BL, Tomasi L et al. Estudo longitudinal da população materno-infantil da região urbana do sul do Brasil, 1993: aspectos metodológicos e resultados preliminares. Rev Saude Publica 1993;30:34-45.

6. Barros FC, Victora CG, editores. Epidemiologia da saúde infantil: um manual para diagnósticos comunitários. São Paulo: Hucitec; 1991. 
7. Monteiro CA. Contribuição para o estudo do significado da evolução do coeficiente de mortalidade infantil no município de São Paulo SP (Brasil) nas três últimas décadas (1950-1979). Rev Saude Publica 1982;16:7-18.

8. Laurenti R, Mello-Jorge MHP, editores. $O$ atestado de óbito. $4^{a}$ ed. São Paulo: Edusp; 2002.

9. Nelson DS, Walsh K, Fleisher GR. Spectrum and frequency of pediatric illness presenting to a general community hospital emergency department. Pediatrics 1992;90:5-10.

10. Burihan PC, Cury MC, Novo NF, Juliano Y, Sigulem DM. Desviantes positivos de saúde para a sobrevivência: um estudo de caso-controle de mortalidade infantil pós-neonatal. Rev Paul Pediatr 2002;20:78-83.

11. Secretaria de Estado da Saúde de São Paulo - SEADE [homepage on the Internet]. Informações de Saúde - Estatísticas vitais - Mortalidade infantil [cited 2008 Feb 12]. Available from: http://www.seade.gov.br/produtos/mortinf/

12. Victora CG, Smith PG, Vaughan JP. Social and environmental influences on child mortality; in Brazil: logistic regression analysis of data from census files. J Biosoc Sci 1986;18:87-101.

13. Whitworth A, Stephenson R. Birth spacing, sibling rivalry and child mortality in India. Soc Sci Med 2002;55:2107-19.
14. Frechette-Piperni S. Loss of a baby and the other children in the family. Soins Pediatr Pueric 2001;199:31-6.

15. Troe EJ, Bos V, Deerenberg IM, Mackenbach JP, Joung IM. Ethnic differences in total and cause-specific infant mortality in The Netherlands. Paediatr Perinat Epidemiol 2006;20:140-7.

16. Markovitz BP, Cook R, Flick LH, Leet TL. Socioeconomic factors and adolescent pregnancy outcomes: distinctions between neonatal and post-neonatal deaths. BMC Public Health 2005;5:79-85.

17. Ribeiro VS, Silva AA, Barbieri MA, Bettiol H, Aragão VM, Coimbra LC et al. Infant mortality: comparison between two birth cohorts from Southeast and Northeast, Brazil. Rev Saude Publica 2004;38:773-9.

18. Currie J, Lin W. Chipping away at health: more on the relationship between income and child health. Health Aff (Millwood) 2007;26:331-44.

19. OMS/UNICEF - Cuidados Primários de Saúde. Declaração de Alma Ata Brasília: Unicef; 1979.

20. Arntzen A, Nybo Andersen AM. Social determinants for infant mortality in the Nordic countries, 1980-2001. Scand J Public Health 2004;32:381-9.

21. Madise NJ, Banda EM, Benaya KW. Infant mortality in Zambia: socioeconomic and demographic correlates. Soc Biol 2003;50:148-66. 